\title{
Bootstrapping Newtonian Gravity
}

\author{
Roberto Casadio ${ }^{a b *}, \quad$ Michele Lenzi ${ }^{a b \dagger}$ and Octavian Micu ${ }^{c \ddagger}$ \\ ${ }^{a}$ Dipartimento di Fisica e Astronomia, Università di Bologna \\ via Irnerio 46, 40126 Bologna, Italy \\ ${ }^{b}$ I.N.F.N., Sezione di Bologna, I.S. FLAG \\ viale B. Pichat 6/2, 40127 Bologna, Italy \\ ${ }^{b}$ Institute of Space Science, Bucharest, Romania \\ P.O. Box MG-23, RO-077125 Bucharest-Magurele, Romania
}

October 23, 2018

\begin{abstract}
A non-linear equation obtained by adding gravitational self-interaction terms to the Poisson equation for Newtonian gravity is here employed in order to analyse a static spherically symmetric homogeneous compact source of given proper mass and radius and the outer vacuum. The main feature of this picture is that, although the freedom of shifting the potential by an arbitrary constant is of course lost, the solutions remain qualitatively very close to the Newtonian behaviour. We also notice that the negative gravitational potential energy is smaller than the proper mass for sources with small compactness, but for sources that should form black holes according to General Relativity, the gravitational potential energy becomes of the same order of magnitude of the proper mass, or even larger. Moreover, the pressure overcomes the energy density for large values of the compactness, but it remains finite for finite compactness, hence there exists no Buchdahl limit. This classical description is meant to serve as the starting point for investigating quantum features of (near) black hole configurations within the corpuscular picture of gravity in future developments.
\end{abstract}

PACS - 04.70.Dy, 04.70.-s, 04.60.-m

\section{Introduction}

It is well-known that Newtonian gravity can be recovered from General Relativity in the weak field and non-relativistic regime [1]. It was also shown long ago that General Relativity is in turn the simplest among the (diffeomorphism invariant) consistent completions of the linear Newtonian gravity with the graviton self-coupling [3]. This can be more easily demostrated in the first order Palatini formalism, in which the metric $g$ and the connection $\Gamma$ are initially treated as independent

\footnotetext{
*E-mail: casadio@bo.infn.it

${ }^{\dagger}$ E-mail: michele.lenzi@studio.unibo.it

${ }_{\ddagger}^{\ddagger}$ E-mail: octavian.micu@spacescience.ro
} 
variables. The relation that makes $\Gamma$ compatible with the metric is then derived and contains the inverse of $g$, which makes it apparent why this completion is essentially non-perturbative. Later on, it was shown [4] that there in fact exists a larger class of Lorentz invariant theories for interacting massless gravitons which are consistent with quantum physics (see also Refs. [5-7]).

In the present work we wish to tackle a much more modest task than recovering classical General Relativity and all of its fundamental symmetries, namely we will study an effective equation for the gravitational potential of a static source which contains a gravitational self-interaction term besides the usual coupling with the matter density. Following an idea from Ref. [8], this equation was derived in details from a Fierz-Pauli Lagrangian in Ref. [9], and it can therefore be viewed as stemming from the truncation of the fully relativistic theory at some "post-Newtonian" order (for the standard post-Newtonian formalism, see Ref. [10]). The main motivation for this study is provided by the quantum model of corpuscular black holes [11], in which the constituents of black holes are assumed to be gravitons marginally bound in their own gravitational potential well. The typical size of this well is given by the characteristic Compton-de Broglie wavelength $\lambda_{\mathrm{G}} \sim R_{\mathrm{H}}$, where $^{1}$

$$
R_{\mathrm{H}}=2 G_{\mathrm{N}} M
$$

is the Schwarzschild (gravitational) radius of the black hole of mass $M$, and whose depth is proportional to the very large number

$$
N_{\mathrm{G}} \sim \frac{M^{2}}{m_{\mathrm{p}}^{2}} \sim \frac{R_{\mathrm{H}}^{2}}{\ell_{\mathrm{p}}^{2}}
$$

of soft quanta in this condensate. When the contribution of gravitons is related to the necessary presence of ordinary matter, the picture appears connected to the post-Newtonian approximation [8]. This can be seen by considering that the (negative) gravitational energy of a source of mass $M$ localised inside a sphere of radius $R$ is given by

$$
U_{\mathrm{N}} \sim M V_{\mathrm{N}} \sim-\frac{G_{\mathrm{N}} M^{2}}{R},
$$

where $V_{\mathrm{N}} \sim-G_{\mathrm{N}} M / R$ is the (negative) Newtonian potential. This potential can be represented by the expectation value of a scalar field over a coherent state $|g\rangle$, whose normalisation then yields the graviton number (1.2), which reproduces Bekenstein's area law [12]. In addition to that, assuming most gravitons have the same wave-length $\lambda_{\mathrm{G}}$, the (negative) energy of each single graviton is correspondingly given by

$$
\epsilon_{\mathrm{G}} \sim \frac{U_{\mathrm{N}}}{N_{\mathrm{G}}} \sim-\frac{m_{\mathrm{p}} \ell_{\mathrm{p}}}{R},
$$

which yields the typical Compton-de Broglie length $\lambda_{\mathrm{G}} \sim R$. The graviton self-interaction energy hence reproduces the (positive) post-Newtonian energy,

$$
U_{\mathrm{GG}}(R) \sim N_{\mathrm{G}} \epsilon_{\mathrm{G}} V_{\mathrm{N}} \sim \frac{G_{\mathrm{N}}^{2} M^{3}}{R^{2}},
$$

\footnotetext{
${ }^{1}$ We shall mostly use units with $c=1$ and the Newton constant $G_{\mathrm{N}}=\ell_{\mathrm{p}} / m_{\mathrm{p}}$, where $\ell_{\mathrm{p}}$ is the Planck length and $m_{\mathrm{p}}$ the Planck mass (so that $\hbar=\ell_{\mathrm{p}} m_{\mathrm{p}}$ ).
} 
and the fact that gravitons in a black hole are marginally bound is reflected by the "maximal packing" condition [11], which roughly reads $U_{\mathrm{N}}+U_{\mathrm{GG}} \simeq 0$ for $R=R_{\mathrm{H}}[8,9,13]$.

The above quantum picture was previously tested by studying small (post-Newtonian) perturbations around the Newtonian potential in Ref. [9]. However, since the post-Newtonian correction $V_{\mathrm{PN}} \sim 1 / r^{2}$ is positive and grows faster than the Newtonian potential near the surface of the source, one is allowed to consider only matter sources with radius $R \gg R_{\mathrm{H}}$ for this approximation to hold. This consistency condition clearly excludes the possibility to study very compact matter sources and, in particular, those on the verge of forming a black hole, that is with $R \simeq R_{\mathrm{H}}$. For the ultimate purpose of including such cases, we shall here study the non-linear equation of the effective theory derived in Ref. [9] at face value, without requiring that the corrections it introduces with respect to the Newtonian potential remain small. We shall nonetheless show that the qualitative behaviour of the complete solutions to our non-linear equation resembles rather closely the Newtonian counterpart. This result, which essentially stems from including a gravitational self-interaction in the Poisson equation, is what we call "bootstrapping" the Newtonian gravity.

The paper is organised as follows: in the next Section we briefly review the derivation of the effective equation for the gravitational potential obtained by including a potential self-interaction; in Section 3, we find the exact solution in the vacuum and, in Section 4, we analyse the case of a homogenous spherical source; a possible connection with the corpuscular model and future perspectives are then discussed in the concluding Section 5.

\section{Effective theory for the gravitational potential}

We shall start by briefly recalling the main steps in the derivation of the non-linear equation which reproduces the Newtonian potential to leading order and includes the effects of gravitational selfinteraction obtained in Ref. [9]. First of all, one assumes the local curvature is small, so that the metric can be written as $g_{\mu \nu}=\eta_{\mu \nu}+h_{\mu \nu}$, where $\eta_{\mu \nu}$ is the flat Minkowski metric with signature $(-,+,+,+)$ and $\left|h_{\mu \nu}\right| \ll 1$. In addition to this weak field limit, we must assume that all matter in the system moves with a characteristic velocity much slower than the speed of light in the (implicitly) chosen reference frame $x^{\mu}=(t, \mathbf{x})$. In fact, we shall just consider (static) spherically symmetric systems, so that $\rho=\rho(r)$ and the only relevant component of the metric is therefore $h_{00}(r) \equiv-2 V(r)$. In this approximation, the Einstein-Hilbert Lagrangian with matter reduces to

$$
L_{\mathrm{N}}[V] \simeq-4 \pi \int_{0}^{\infty} r^{2} \mathrm{~d} r\left[\frac{\left(V^{\prime}\right)^{2}}{8 \pi G_{\mathrm{N}}}+\rho V\right],
$$

where $f^{\prime} \equiv \mathrm{d} f / \mathrm{d} r$. The corresponding equation of motion is just the Poisson equation,

$$
r^{-2}\left(r^{2} V^{\prime}\right)^{\prime} \equiv \triangle V=4 \pi G_{\mathrm{N}} \rho,
$$

for the Newtonian potential $V=V_{\mathrm{N}}$.

In order to go beyond the Newtonian approximation, we modify the latter functional by adding a non-linear term. This term can be obtained by noting that the Hamiltonian

$$
H_{\mathrm{N}}[V]=-L_{\mathrm{N}}[V]=4 \pi \int_{0}^{\infty} r^{2} \mathrm{~d} r\left(-\frac{V \triangle V}{8 \pi G_{\mathrm{N}}}+\rho V\right),
$$


computed on-shell by means of Eq. (2.2), yields the Newtonian potential energy

$$
\begin{aligned}
U_{\mathrm{N}}(r) & =2 \pi \int_{0}^{r} \bar{r}^{2} \mathrm{~d} \bar{r} \rho(\bar{r}) V(\bar{r}) \\
& =\frac{1}{2 G_{\mathrm{N}}} \int_{0}^{r} \bar{r}^{2} \mathrm{~d} \bar{r} V(\bar{r}) \triangle V(\bar{r}) \\
& =-\frac{1}{2 G_{\mathrm{N}}} \int_{0}^{r} \bar{r}^{2} \mathrm{~d} \bar{r}\left[V^{\prime}(\bar{r})\right]^{2},
\end{aligned}
$$

where we used Eq. (2.2) and then integrated by parts discarding boundary terms. One can view the above $U_{\mathrm{N}}$ as given by the interaction of the matter distribution enclosed in a sphere of radius $r$ with the gravitational field. Following Ref. [8] (see also Ref. [14]), we then define a self-gravitational source $J_{V}$ proportional to the gravitational energy $U_{\mathrm{N}}$ per unit volume, that is [9]

$$
J_{V}(r)=\frac{4}{4 \pi r^{2}} \frac{\mathrm{d}}{\mathrm{d} r} U_{\mathrm{N}}(r)=-\frac{1}{2 \pi G_{\mathrm{N}}}\left[V^{\prime}(r)\right]^{2} .
$$

Upon including this new source term, and the analogous higher order term $J_{\rho}=-2 V^{2}$ which couples with the matter source, we obtain the total Lagrangian [9]

$$
\begin{aligned}
L[V] & =L_{\mathrm{N}}[V]-4 \pi \int_{0}^{\infty} r^{2} \mathrm{~d} r\left(q_{\Phi} J_{V} V+q_{\Phi} J_{\rho} \rho\right) \\
& =-4 \pi \int_{0}^{\infty} r^{2} \mathrm{~d} r\left[\frac{\left(V^{\prime}\right)^{2}}{8 \pi G_{\mathrm{N}}}\left(1-4 q_{\Phi} V\right)+\rho V\left(1-2 q_{\Phi} V\right)\right] .
\end{aligned}
$$

Finally, the Euler-Lagrange equation for $V$ is thus given by

$$
\left(1-4 q_{\Phi} V\right)\left(\triangle V-4 \pi G_{\mathrm{N}} \rho\right)=2 q_{\Phi}\left(V^{\prime}\right)^{2},
$$

where $q_{\Phi}$ is a self-coupling parameter. In particular, it was shown in Ref. [9] that the first postNewtonian expansion of the Schwarzschild metric is recovered for $q_{\Phi}=1$, and we shall therefore assume this value in the following (we will briefly come back to this point in the Conclusions).

In the next sections, we shall analyse Eq. (2.7) as an effective description of the static gravitational field $V$ generated by a static source of density $\rho$ in flat space-time. In other words, we abandon, or disregard, its geometric origin given by the Einstein-Hilbert action and proceed by assuming there exists a reference frame in which the motion of test particles are described by Newton's law with a potential that solves Eq. (2.7). Before we try and solve this equation, it is then important to note that the freedom to shift the Newtonian potential by an arbitrary constant, say $V_{0}$, is now lost in general. In fact, if $V_{\mathrm{c}}$ solves Eq. (2.7), for any $\bar{V}=V_{\mathrm{c}}+V_{0}$ one finds

$$
\left[1-4\left(V_{c}+V_{0}\right)\right]\left(\triangle V_{c}-4 \pi G_{\mathrm{N}} \rho\right)-2\left(V_{\mathrm{c}}^{\prime}\right)^{2}=-4 V_{0}\left(\triangle V_{\mathrm{c}}-4 \pi G_{\mathrm{N}} \rho\right),
$$

which therefore means that $\bar{V}$ would still be a solution only if $V_{\mathrm{c}}=V_{\mathrm{N}}$. This property clearly parallels General Relativity. We however note that Eq. (2.6) does not yet contain the pressure as a source, which will have to be added in order to ensure energy conservation in general, as we will comment more extensively in Section 5.

On a qualitative ground, one might expect that the term in the r.h.s. of Eq. (2.7) becomes less important for $V_{\mathrm{c}}$ negative and large, and one approximately recovers the Poisson equation (2.2) in this case. We shall in fact see that the solution to Eq. (2.7) can be conveniently expressed as a (somehow small) perturbation about the Newtonian potential where $\rho \neq 0$. On the other hand, in the vacuum $\rho=0$ and the effect of the new gravitational self-coupling in Eq. (2.7) leads to (possibly) significant departures from the Newtonian behaviour. 

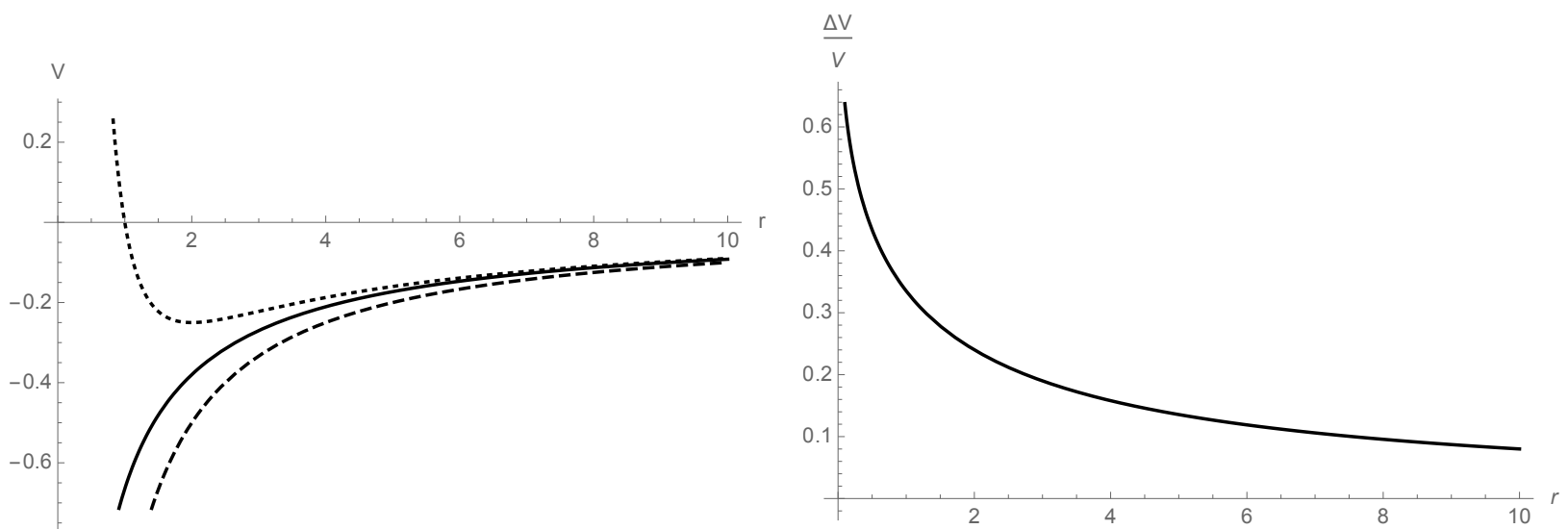

Figure 1: Left panel: potential $V_{\mathrm{c}}$ (solid line) vs Newtonian potential (dashed line) vs order $G_{\mathrm{N}}^{2}$ expansion of $V_{\mathrm{c}}$ (dotted line) for $r>0$. Right panel: relative difference $\left(V_{\mathrm{N}}-V_{\mathrm{c}}\right) / V_{\mathrm{N}}$ for $r>0$ (all quantities are in units of $\left.G_{\mathrm{N}} M\right)$.

\section{Spherical vacuum solution}

In the vacuum, where $\rho=0$, Eq. (2.7) with $q_{\Phi}=1$ reads

$$
\triangle V=\frac{2\left(V^{\prime}\right)^{2}}{1-4 V}
$$

which is exactly solved by

$$
V_{\mathrm{c}}=\frac{1}{4}\left[1-c_{1}\left(1+\frac{c_{2}}{r}\right)^{2 / 3}\right]
$$

where $c_{1}$ and $c_{2}$ are integration constants, and we note again that that one cannot shift $V_{\mathrm{c}}$ by an arbitrary constant $V_{0}$.

The two integration constants can still be fixed by requiring the expected Newtonian behaviour in terms of the ADM mass [15] $M$ for large $r$. One must then have $c_{1}=1$ and $c_{2}=6 G_{\mathrm{N}} M$, which yield

$$
V_{\mathrm{c}}=\frac{1}{4}\left[1-\left(1+\frac{6 G_{\mathrm{N}} M}{r}\right)^{2 / 3}\right] .
$$

The large $r$ expansion now reads

$$
V_{\mathrm{c}} \underset{r \rightarrow \infty}{\stackrel{\sim}{\rightrightarrows}}-\frac{G_{\mathrm{N}} M}{r}+\frac{G_{\mathrm{N}}^{2} M^{2}}{r^{2}}-\frac{8 G_{\mathrm{N}}^{3} M^{3}}{3 r^{3}}
$$

and contains the expected post-Newtonian term $V_{\mathrm{PN}}$ of order $G_{\mathrm{N}}^{2}$ without any further assumptions [9]. Moreover, unlike its truncation at order $G_{\mathrm{N}}^{2}$, the above $V_{\mathrm{c}}$ tracks the Newtonian solution for all values $r>0$ (see Fig. 1). In particular, $V_{\mathrm{c}}$ remains (increasingly) negative like $V_{\mathrm{N}}$, but diverges slower than $V_{\mathrm{N}}$ for $r \rightarrow 0$, since

$$
\frac{V_{\mathrm{c}}}{V_{\mathrm{N}}} \underset{r \rightarrow 0}{\sim}\left(\frac{r}{G_{\mathrm{N}} M}\right)^{1 / 3}
$$


as is also displayed by the relative difference in the right panel of Fig. 1. This shows that the added source in the r.h.s. of Eq. (3.1) acts as a (partial) regulator. In Fig. 1, we also plot the large $r$ expansion (3.4) up to the first post-Newtonian approximation of order $G_{\mathrm{N}}^{2}$, which describes a repulsive force for $r<2 G_{\mathrm{N}} M$. Of course, gravity is attractive and one must view this result as indicating the post-Newtonian expansion fails at such short distances.

\section{Homogeneous ball in vacuum}

Since we are interested in compact sources, we will consider the simplest case in which the matter density is homogeneous and vanishes outside the sphere of radius $r=R$, that is

$$
\rho=\frac{3 M_{0}}{4 \pi R^{3}} \Theta(R-r),
$$

where $\Theta$ is the Heaviside step function, and

$$
M_{0}=4 \pi \int_{0}^{R} r^{2} \mathrm{~d} r \rho(r) .
$$

Even with this static and very simple matter density, it is impossible to find analytic solutions to Eq. (2.7), so we shall first solve it numerically and then proceed to find analytical approximations.

For the numerical solutions, it is useful to introduce dimensionless units by considering the size $R$ of the source as the reference length, that is we define

$$
r \equiv R \tilde{r}, \quad G_{\mathrm{N}} M_{0} \equiv R \tilde{M}_{0},
$$

so that Eq. (2.7) for $\tilde{V}=V(\tilde{r})$ reads

$$
(1-4 \tilde{V})(\tilde{\triangle} \tilde{V}-4 \pi \tilde{\rho})=2\left(\tilde{V}^{\prime}\right)^{2},
$$

where $\tilde{f}^{\prime} \equiv \mathrm{d} \tilde{f} / \mathrm{d} \tilde{r}$, the operator $\tilde{\triangle}=\tilde{r}^{-2} \partial_{\tilde{r}}\left(\tilde{r}^{2} \partial_{\tilde{r}}\right)$ and

$$
\tilde{\rho}=R^{2} G_{\mathrm{N}} \rho \equiv \frac{3 \tilde{M}_{0}}{4 \pi} \Theta(1-\tilde{r}) .
$$

\subsection{Newtonian solution}

As a reference, we shall first consider the Newtonian potential for this homogeneous sphere, and then analyse the different regions of space separately for the complete Eq. (2.7).

Eq. (2.2) with the density (4.1) in dimensionless units reads

$$
\tilde{\triangle} \tilde{V}=3 \tilde{M}_{0} \Theta(1-\tilde{r}) .
$$

Its complete solution can be easily obtained by matching the (asymptotically regular) vacuum solution for $\tilde{r}>1$,

$$
\tilde{V}_{\mathrm{N}}^{+}=-\frac{\tilde{M}}{\tilde{r}}
$$


where $G_{\mathrm{N}} M \equiv R \tilde{M}$, with the solution (regular in the centre) for $0 \leq \tilde{r}<1$,

$$
\tilde{V}_{\mathrm{N}}^{-}=\frac{\tilde{M}_{0}}{2}\left(\tilde{r}^{2}-3 C\right) \text {, }
$$

where $C$ is a constant to be determined. In particular, requiring that both $\tilde{V}_{\mathrm{N}}$ and its derivative $\tilde{V}_{\mathrm{N}}^{\prime}$ be continuous across $\tilde{r}=1$ fixes the integration constant $C=1$ and $\tilde{M}=\tilde{M}_{0}$, that is

$$
\tilde{V}_{\mathrm{N}}= \begin{cases}\frac{\tilde{M}_{0}}{2}\left(\tilde{r}^{2}-3\right) & \text { for } \quad 0 \leq \tilde{r}<1 \\ -\frac{\tilde{M}_{0}}{\tilde{r}} & \text { for } \quad \tilde{r}>1 .\end{cases}
$$

\subsection{Fully numerical solutions}

The above Newtonian solution $\tilde{V}_{\mathrm{N}}$ is obtained from the boundary conditions

$$
\left\{\begin{array}{c}
\tilde{V}(\tilde{r}) \underset{\tilde{r} \rightarrow \infty}{\rightarrow} 0 \\
\tilde{V}^{\prime}(0)=0,
\end{array}\right.
$$

which fix all of the integration constants and only leave a dependence on $\tilde{M}_{0}=\tilde{M}$. The same boundary conditions are then required for the solution $\tilde{V}_{\text {c }}$ to Eq. (4.4), which we therefore expect will be uniquely determined by $\tilde{M}_{0}$.

Some numerical solutions $\tilde{V}_{\text {c }}$ computed for given values of $\tilde{M}_{0}$ are shown in Fig. 2, where they are also compared with the corresponding Newtonian potentials (4.9). The main features of the numerical solutions $\tilde{V}_{\mathrm{c}}$ are that they systematically lie below their Newtonian counterparts $\tilde{V}_{\mathrm{N}}$, but their shapes are qualitatively very close.

\subsection{Analytical approximations}

The features found numerically will now guide us to find analytical approximations for the interior of the homogeneous source. First of all, outside the source $\rho=0$, and the exact solution (3.3) in dimensionless units reads

$$
\tilde{V}_{\mathrm{c}}=\frac{1}{4}\left[1-\left(1+\frac{6 \tilde{M}}{\tilde{r}}\right)^{2 / 3}\right],
$$

In the following, it will be also useful to consider the value of this potential at $\tilde{r}=1$,

$$
\tilde{V}_{\mathrm{c}}^{+}=\frac{1}{4}\left[1-(1+6 \tilde{M})^{2 / 3}\right],
$$

and of its derivative,

$$
\tilde{V}_{\mathrm{c}}^{\prime+}=\tilde{M}(1+6 \tilde{M})^{-1 / 3}=\tilde{V}_{\mathrm{N}}^{\prime+}(1+6 \tilde{M})^{-1 / 3}
$$

where $\tilde{V}_{\mathrm{N}}$ is the Newtonian potential, and the term in brackets contains the corrections to the Newtonian force at the surface of the sphere. 

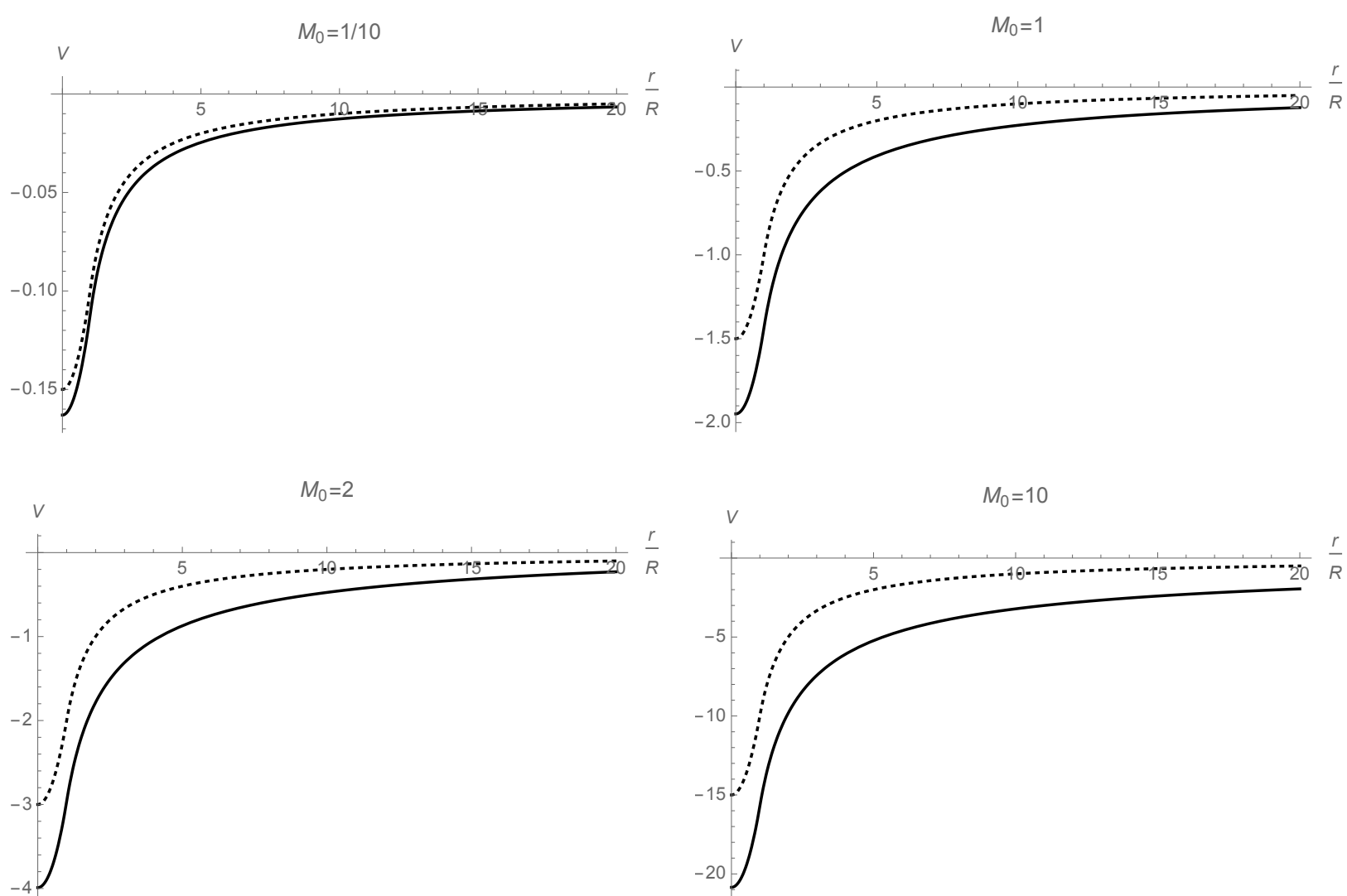

Figure 2: Numerical solution $\tilde{V}_{\mathrm{c}}$ (solid line) vs Newtonian potential $\tilde{V}_{\mathrm{N}}$ (dashed line) for $\tilde{M}_{0}=1 / 10$ (top left), $\tilde{M}_{0}=1$ (top right), $\tilde{M}_{0}=2$ (bottom left) and $\tilde{M}_{0}=10$ (bottom right).

\subsubsection{Inside the homogeneous source}

Let us next consider the interior of the matter source, that is $0 \leq \tilde{r}<1$. An exact solution for the homogeneous density can be found which is however positive everywhere and cannot be used for describing a ball immersed in an outer vacuum (see Appendix C).

Since it is hard to find exact analytical solutions where the density $\rho>0$, we first write

$$
\tilde{V}_{\mathrm{c}}=\tilde{V}_{\mathrm{N}}+\tilde{W},
$$

and assume $|\tilde{W}| \ll\left|\tilde{V}_{\mathrm{N}}\right|$ for $\tilde{r} \simeq 0$. Upon replacing into Eq. (4.4), we obtain

$$
\tilde{\triangle} \tilde{W}=\frac{2\left(\tilde{V}_{\mathrm{N}}^{\prime}+\tilde{W}^{\prime}\right)^{2}}{1-4 \tilde{V}_{\mathrm{N}}-4 \tilde{W}} \simeq \frac{2\left(\tilde{V}_{\mathrm{N}}^{\prime}\right)^{2}}{1-4 \tilde{V}_{\mathrm{N}}},
$$

where $\tilde{V}_{\mathrm{N}}$ is given in Eq. (4.8), with $C$ arbitrary. The solution around $\tilde{r}=0$ can be written as

$$
\tilde{W} \simeq \frac{\tilde{M}_{0}^{2} \tilde{r}^{4}}{10\left(1+6 C \tilde{M}_{0}\right)}\left[1+\frac{20 \tilde{M}_{0} \tilde{r}^{2}}{21\left(1+6 C \tilde{M}_{0}\right)}\right] \equiv \tilde{W}_{4}+\tilde{W}_{6}
$$



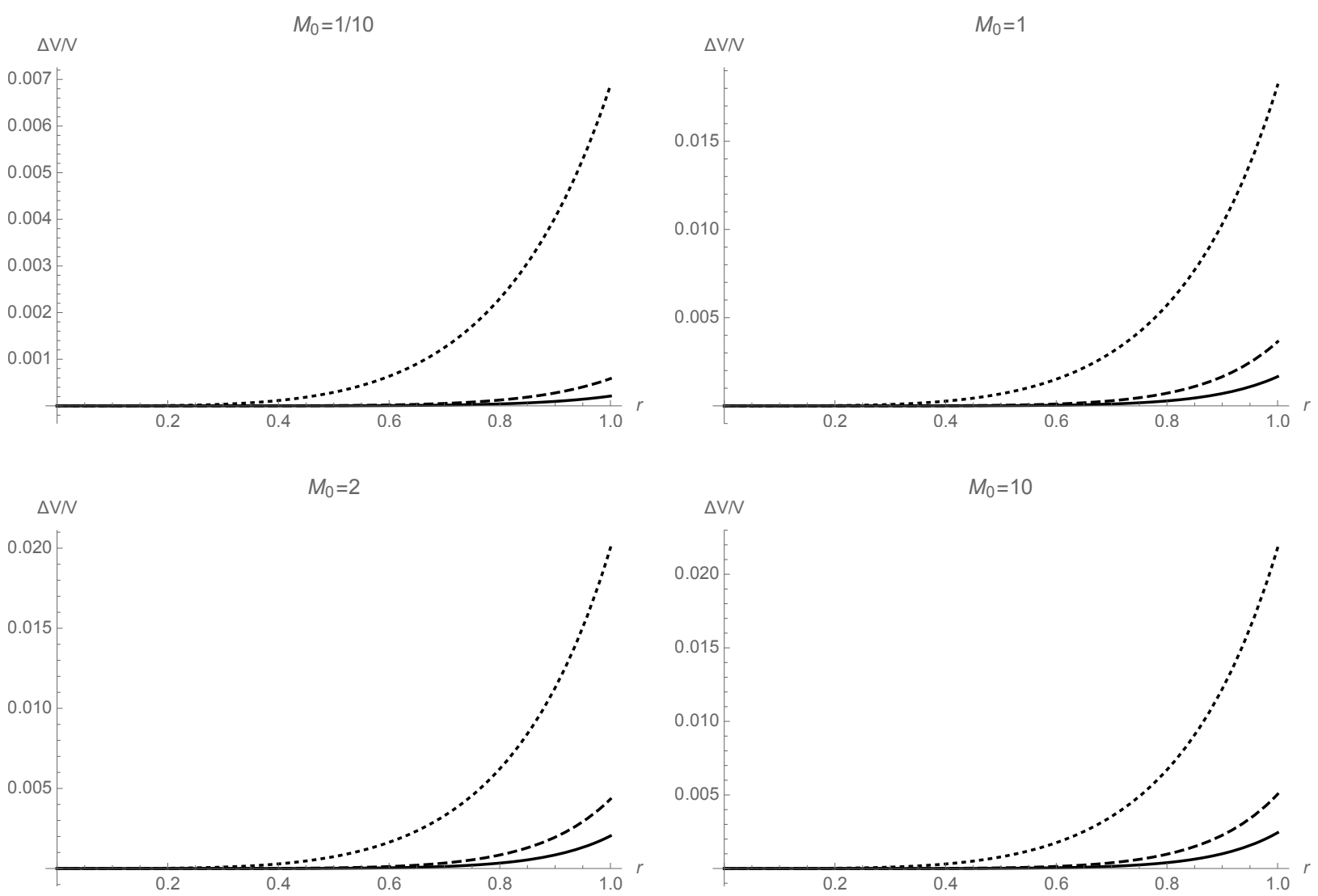

Figure 3: Relative error $\left(\tilde{V}_{\mathrm{c}}-\tilde{V}_{\mathrm{n}}\right) / \tilde{V}_{\mathrm{n}}$ with respect to the numerical solution $\tilde{V}_{\mathrm{n}}$ for $\tilde{V}_{\mathrm{c}}=\tilde{V}_{\mathrm{N}}+\tilde{W}_{4}+\tilde{W}_{6}$ (solid line) vs $\tilde{V}_{\mathrm{c}}=\tilde{V}_{\mathrm{N}}+\tilde{W}_{4}$ (dashed line) vs Newtonian case $\tilde{V}_{\mathrm{c}}=\tilde{V}_{\mathrm{N}}$ (dotted line) for $C=1$ and $\tilde{M}_{0}=1 / 10$ (top left), $\tilde{M}_{0}=1$ (top right), $\tilde{M}_{0}=2$ (bottom left) and $\tilde{M}_{0}=10$ (bottom right).

so that

$$
\left|\frac{\tilde{W}_{4}}{\tilde{V}_{\mathrm{N}}}\right| \leq \frac{\tilde{M}_{0}}{15 C\left(1+6 C \tilde{M}_{0}\right)}
$$

where we assumed $C>1$, since the numerical solutions suggest that $\left|\tilde{V}_{\mathrm{c}}(0)\right|>\left|\tilde{V}_{\mathrm{N}}(0)\right|$. We then find our approximation should be rather accurate for any values of $\tilde{M}_{0}$ as long as $C \geq 1$.

The accuracy of the above analytical results is compared with the numerical solutions $\tilde{V}_{\mathrm{n}}$ in Fig. 3, which again shows that the extra source in the r.h.s. of Eq. (2.7) acts as a regulator, and whose effects are significantly dumped where $\rho>0$. It is important to remark that the above expansion about the Newtonian potential $\tilde{V}_{\mathrm{N}}$ holds for any value of $\tilde{V}_{\mathrm{N}}(0)$, which is determined by the constant $C$ in Eq. (4.8). Since the latter is arbitrary, and can only be fixed by matching with the outer Newtonian potential, the above results show that we can in fact have a solution $\tilde{V}_{\mathrm{c}}$ with $\tilde{V}_{\mathrm{c}}^{\prime}(0)=0$ and $\tilde{V}_{\mathrm{c}}(0)$ such that it will match the outer solution $(3.3)$ at $\tilde{r}=1$. 

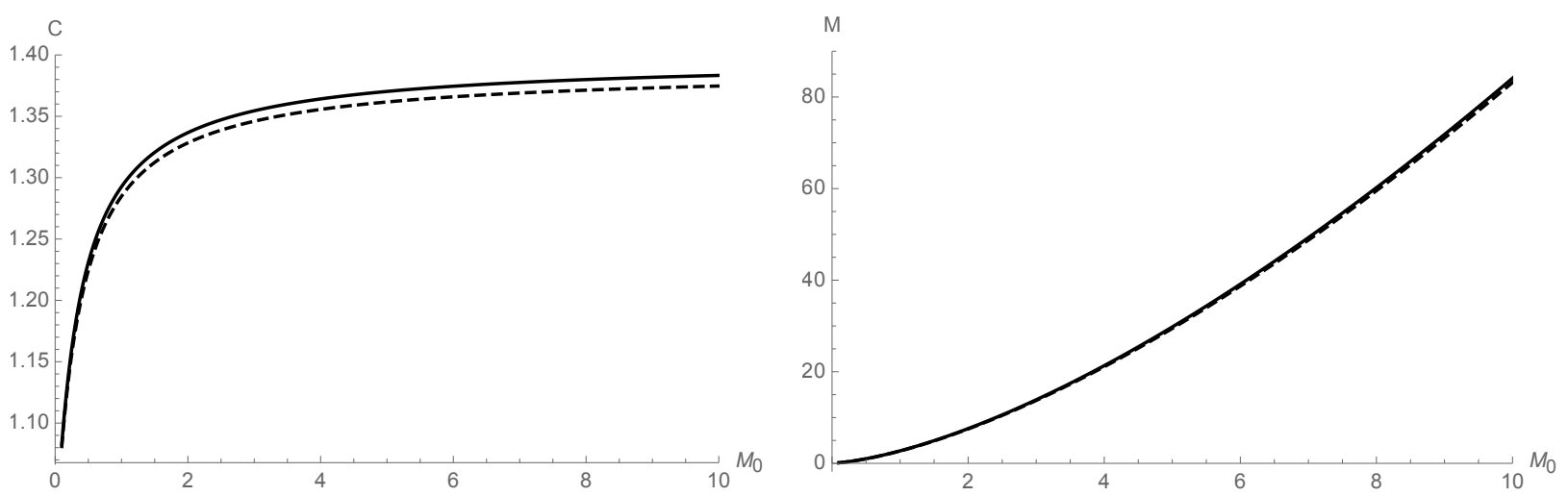

Figure 4: Ratio $C=\tilde{V}_{\mathrm{c}} / \tilde{V}_{\mathrm{N}}$ (left panel) and mass $\tilde{M}$ (right panel) computed numerically using $\tilde{V}_{\mathrm{c}}=\tilde{V}_{\mathrm{N}}+\tilde{W}_{4}+\tilde{W}_{6}$ (solid lines) and using $\tilde{V}_{\mathrm{c}}=\tilde{V}_{\mathrm{N}}+\tilde{W}_{4}$ (dashed lines).

\subsubsection{Matching at the surface}

In order to determine the potential for all values of $\tilde{r}>0$, we can start from the approximate solution (4.14) for the interior of the source and match it with the exact outer solution (4.11), at $\tilde{r}=1$ (corresponding to $r=R$ ). In particular, with $\tilde{V}_{\mathrm{N}}$ in Eq. (4.8) and $\tilde{W}$ in Eq. (4.16), continuity of the potential and of its derivative at $\tilde{r}=1$ (see Appendix B) results in the two conditions

$$
\left\{\begin{array}{l}
\tilde{V}_{\mathrm{N}}^{-}+\tilde{W}^{-}=\tilde{V}_{\mathrm{c}}^{+} \\
\tilde{V}_{\mathrm{N}}^{\prime-}+\tilde{W}^{\prime-}=\tilde{V}_{\mathrm{c}}^{\prime+}
\end{array}\right.
$$

for the three parameters $\tilde{M}_{0}, \tilde{M}$ and $C$. We can thus determine $C$ and $\tilde{M}$ in terms of $\tilde{M}_{0}$. The numerical solutions for $C$ and $\tilde{M}$ in the range $1 / 10 \leq \tilde{M}_{0} \leq 10$ are shown in Fig. 4 .

Since neglecting $\tilde{W}_{6}$ does not introduce a large error, we can estimate $C$ and $\tilde{M}$ analytically just using $\tilde{W} \simeq \tilde{W}_{4}$, so that continuity of the potential at $\tilde{r}=1$ reads

$$
2 \tilde{M}_{0}(1-3 C)+\frac{2 \tilde{M}_{0}^{2}}{5\left(1+6 C \tilde{M}_{0}\right)} \simeq 1-(1+6 \tilde{M})^{2 / 3},
$$

whereas continuity of the derivative of the potential at $\tilde{r}=1$ requires

$$
\tilde{M}_{0}+\frac{2 \tilde{M}_{0}^{2}}{5\left(1+6 C \tilde{M}_{0}\right)} \simeq \frac{\tilde{M}}{(1+6 \tilde{M})^{1 / 3}} .
$$

For $\tilde{M}_{0} \ll 1$, Eq. (4.20) yields the same result of Ref. [9], to wit

$$
\tilde{M} \simeq \tilde{M}_{0}\left(1+\frac{12}{5} \tilde{M}_{0}\right),
$$

and from Eq. (4.19) one then finds

$$
C \simeq 1+\tilde{M}_{0},
$$


which reproduce the Newtonian solution $C \simeq 1$ and $\tilde{M} \simeq \tilde{M}_{0}$ at lowest order, and are in agreement with the numerical solutions (see, e.g. the top left panel of Fig. 2). In the opposite limit $\tilde{M}_{0} \gg 1$, Eq. (4.20) yields

$$
\tilde{M} \simeq \sqrt{6} \tilde{M}_{0}^{3 / 2}
$$

and Eq. (4.19) gives the asymptotic value

$$
C=\frac{\tilde{V}_{\mathrm{c}}(0)}{\tilde{V}_{\mathrm{N}}(0)} \simeq 1.34
$$

From the left panel of Fig. 4, we see that the above estimate of $C$ is just a bit short of the value

$$
C \simeq 1.4
$$

obtained from solving Eqs. (4.4) numerically for $\tilde{M}_{0} \simeq 1000$. This behaviour of $C$ and $\tilde{M}$ is also in agreement with the fully numerical solutions described in Section 4.2.

It is now important to recall that $\tilde{M}_{0}=G_{\mathrm{N}} M_{0} / R \gg 1$ corresponds to a source with very large density, asymptotically approaching a Dirac delta function. On the opposite, $\tilde{M}_{0}=G_{\mathrm{N}} M_{0} / R \ll 1$ represents a source with small density for which one expects the weak field approximation holds. In the next Section, we will specifically look at this case.

\subsection{Weak field regime}

The above picture simplifies significantly in the weak field regime, which was already studied in Ref. [9]. In fact Eq. (4.4) can be approximated for $|\tilde{V}| \ll 1$ as

$$
\tilde{\triangle} \tilde{V}_{\mathrm{WF}} \simeq 4 \pi \tilde{\rho}+2\left(\tilde{V}_{\mathrm{WF}}^{\prime}\right)^{2}
$$

and the main feature of this equation is that one regains the freedom to shift the potential by an arbitrary constant, like in purely Newtonian gravity.

In the vacuum, Eq. (4.26) reads

$$
\tilde{\triangle} \tilde{V}_{\mathrm{WF}} \simeq 2\left(\tilde{V}_{\mathrm{WF}}^{\prime}\right)^{2}
$$

and is exactly solved by

$$
\tilde{V}_{\mathrm{WF}}=-\frac{1}{2} \ln \left(1+\frac{2 \tilde{M}}{\tilde{r}}\right),
$$

in which two arbitrary integration constants were fixed again by requiring the expected Newtonian behaviour in terms of the ADM mass $\tilde{M}$ for large $\tilde{r}$. The large $r$ expansion of the above solution is the same as Eq. (3.4) up to, and including order $G_{\mathrm{N}}^{2}$ and the correct post-Newtonian term is again recovered without any further assumptions. In Fig. 5, we plot the weak field solution (4.28) and compare it to the exact non-linear solution (3.3) and the Newtonian potential for the same value of the ADM mass. Clearly, the plot is only meaningful for $r \gg G_{\mathrm{N}} M=1$ (in the units of the graph), since the condition $\left|\tilde{V}_{\mathrm{WF}}\right| \ll 1$ implies that $\tilde{r} \gg 2 \tilde{M}$. 


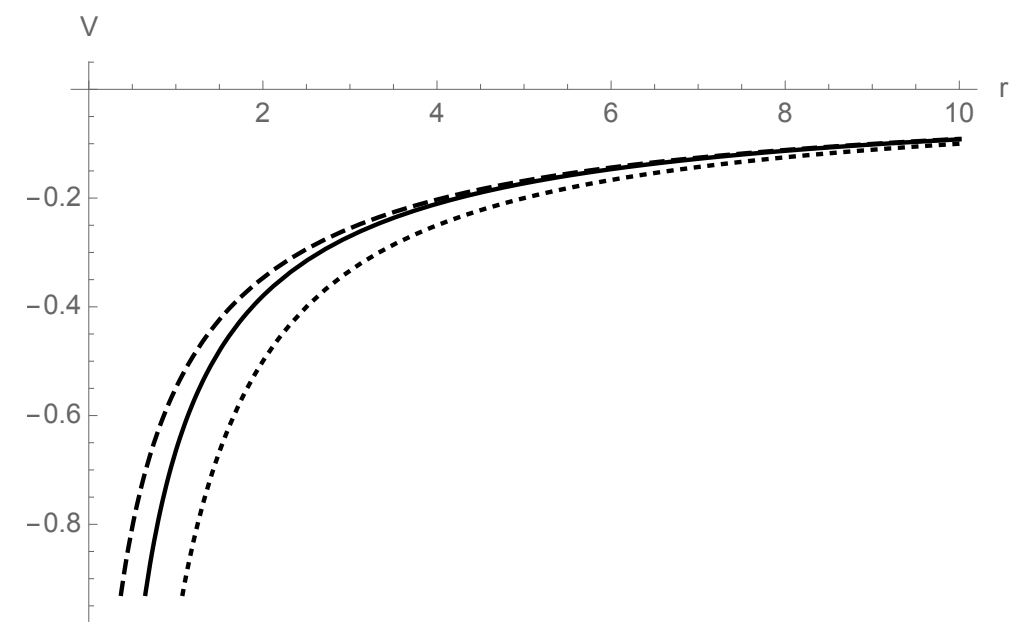

Figure 5: Potential $V_{\mathrm{WF}}$ (dashed line) vs $V_{\mathrm{c}}$ (solid line) vs Newtonian potential (dotted line) in units of $G_{\mathrm{N}} M$.

The interior of a homogeneous source is now approximately described by the equation

$$
\tilde{\triangle} \tilde{V}_{\mathrm{WF}} \simeq 3 \tilde{M}_{0}+2\left(\tilde{V}_{\mathrm{WF}}^{\prime}\right)^{2}
$$

which is solved exactly by

$$
\tilde{V}_{\mathrm{WF}}=A-\frac{1}{2} \ln \left[\frac{\sin \left(B+\sqrt{6 \tilde{M}_{0}} \tilde{r}\right)}{\tilde{r}}\right],
$$

where $0 \leq \tilde{r} \leq 1$ and $\tilde{M}_{0} \ll 1$ in order to preserve the weak field approximation. Regularity in $\tilde{r}=0$ then fixes the integration constant $B=0$ and, upon defining $A-\ln \left(6 \tilde{M}_{0}\right) / 4 \equiv 3 \tilde{M}_{0} C / 2$, one finds

$$
\tilde{V}_{\mathrm{WF}}=A-\frac{1}{2} \ln \left[\frac{\sin \left(\sqrt{6 \tilde{M}_{0}} r\right)}{\tilde{r}}\right] \simeq \frac{\tilde{M}_{0}}{2}\left(\tilde{r}^{2}-3 C\right)+\frac{\tilde{M}_{0}^{2}}{10} \tilde{r}^{4}
$$

which indeed agrees with the limit $\tilde{M}_{0} \ll 1$ of Eq. (4.16).

Continuity of the derivative of the potential across $\tilde{r}=1$ reads

$$
\tilde{M}_{0}\left(1+\frac{2 \tilde{M}_{0}}{5}\right) \simeq \tilde{M}(1-2 \tilde{M})
$$

and is solved by Eq. (4.21). Upon using this relation between $\tilde{M}$ and $\tilde{M}_{0}$, continuity of the potential at $\tilde{r}=1$ reads

$$
\frac{3 \tilde{M}_{0}}{2}(C-1) \simeq \frac{3 \tilde{M}_{0}^{2}}{2}
$$

which is again solved by Eq. (4.22). This shows that for $\tilde{M}_{0} \ll 1$, one can just employ the much simpler Eq. (4.26), like it was done in Ref. [9]. 


\subsection{Pressure and stability}

So far we completely neglected the pressure required to have a static configuration at fixed radius $R$. In the following, we will employ a Newtonian argument in order to estimate the necessary pressure and deduce the corresponding potential energy contribution in a way so as to support the identification of $M$ as the total energy of the system.

\subsubsection{Newtonian pressure}

It is well know from the Newtonian theory that the condition of hydrostatic equilibrium for the pressure $p_{\mathrm{N}}$ reads [1]

$$
p_{\mathrm{N}}^{\prime}(r)=-V_{\mathrm{N}}^{\prime}(r) \rho(r)=-\frac{G_{\mathrm{N}} m(r)}{r^{2}} \rho(r)
$$

where $m=m(r)$ is the mass contained within a sphere of radius $r$ defined in Eq. (D.2). This equation can be easily solved for the homogeneous density (4.1) generating the Newtonian potential (4.9) inside the source. Upon requiring that the pressure vanishes at the surface, $p_{\mathrm{N}}(R)=0$, the solution reads

$$
p_{\mathrm{N}}(r)=\frac{3 G_{\mathrm{N}} M_{0}^{2}\left(r^{2}-R^{2}\right)}{8 \pi R^{6}} .
$$

It is then straightforward to calculate the associated (Newtonian) potential energy as

$$
\begin{aligned}
U_{\mathrm{BN}}(r) & =B_{\mathrm{N}}-4 \pi \int_{0}^{r} \mathrm{~d} \bar{r} \bar{r}^{2} p_{\mathrm{N}}(\bar{r}) \\
& =B_{\mathrm{N}}+\frac{G_{\mathrm{N}} M_{0}^{2}\left(3 r^{2}-5 R^{2}\right) r^{3}}{10 R^{6}},
\end{aligned}
$$

where $B_{\mathrm{N}}$ is an arbitrary integration constant.

The constant $B_{\mathrm{N}}$ can in fact depend on the parameters of our model, like $M_{0}$ and $R$. In particular, the condition of stability requires that the work done by the pressure $p_{\mathrm{N}}$ cancels against the work done by the gravitational force for an infinitesimal change in the radius $R$, that is

$$
\frac{\mathrm{d} U_{\mathrm{BN}}}{\mathrm{d} R}=-\frac{\mathrm{d} U_{\mathrm{N}}}{\mathrm{d} R}=-\frac{3 G_{\mathrm{N}} M_{0}^{2}}{5 R^{2}},
$$

in which we used Eq. (A.3) for the Newtonian potential energy $U_{\mathrm{N}}=U_{\mathrm{N}}(R)$. This condition yields

$$
B_{\mathrm{N}}\left(M_{0}, R\right)=\frac{4 G_{\mathrm{N}} M_{0}^{2}}{5 R},
$$

and

$$
U_{\mathrm{BN}}(r)=\frac{G_{\mathrm{N}} M_{0}^{2}\left(3 r^{5}-5 r^{3} R^{2}+8 R^{5}\right)}{10 R^{6}},
$$

which ensures that the total energy $E$ of the system

$$
E=M_{0}+U_{\mathrm{BN}}(R)+U_{\mathrm{N}}(R)=M_{0} .
$$

Moreover, it is worth recalling that $M_{0}=M$ at the Newtonian level, so that $E=M$. 

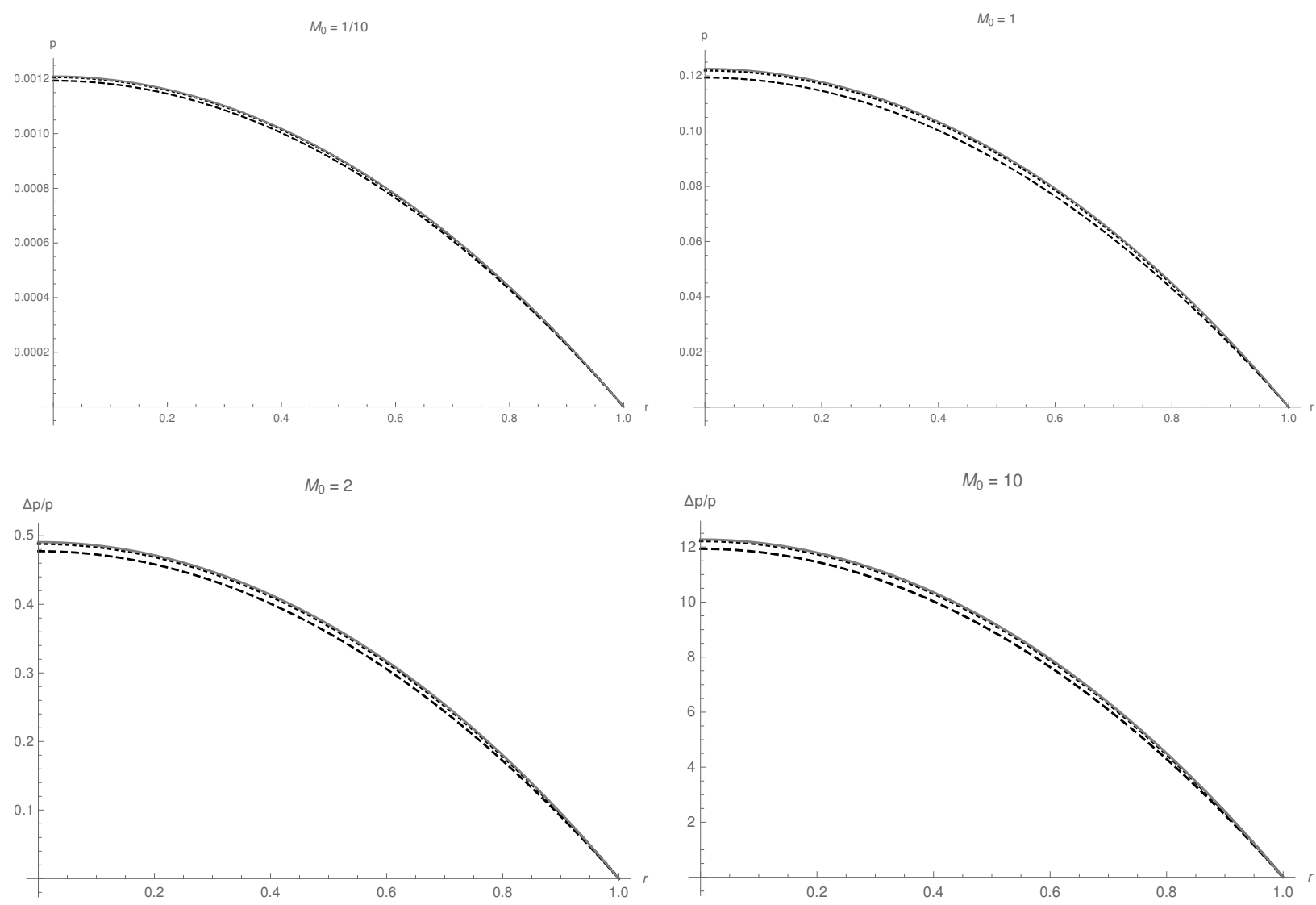

Figure 6: Numerical solution (solid gray line) vs analytical approximation (4.42) with $C=1.34$ (dotted line) vs Newtonian pressure (4.35) (dashed line) for $\tilde{M}_{0}=1 / 10$ (top left), $\tilde{M}_{0}=1$ (top right), $\tilde{M}_{0}=2$ (bottom left) and $\tilde{M}_{0}=10$ (bottom right).

\subsubsection{Post-Newtonian pressure}

For the configurations found in Section 4, we follow the same line of reasoning and simply replace the Newtonian potential in Eq. (4.34) with the solution $V_{\mathrm{c}}$, to wit

$$
p^{\prime}(r)=-V_{\mathrm{c}}^{\prime}(r) \rho(r) .
$$

For the potential $V_{\mathrm{c}}$, we shall employ the analytical approximation (4.14) up to the term $W_{4}$ in Eq. (4.16), for which Eq. (4.41) can be solved to obtain

$$
p(r)=\frac{3 G_{\mathrm{N}} M_{0}^{2}\left(R^{2}-r^{2}\right)\left\{5 R^{3}+G_{\mathrm{N}} M_{0}\left[r^{2}+(1+30 C) R^{2}\right]\right\}}{40 \pi R^{8}\left(R+6 C G_{\mathrm{N}} M_{0}\right)},
$$

which is very close to the fully numerical result, as shown in Fig. 6.

Like in the Newtonian approximation, upon integrating the above pressure on the volume of the source, we can obtain the baryonic potential energy $U_{\mathrm{B}}$ necessary for the compact object to be in mechanical equilibrium,

$$
\begin{aligned}
U_{\mathrm{B}}(r)= & \frac{G_{\mathrm{N}} M_{0}^{2}\left\{3 G_{\mathrm{N}} M_{0} r^{7}+21 r^{5} R^{2}\left(R+6 C G_{\mathrm{N}} M_{0}\right)-7 r^{3} R^{4}\left[5 R+G_{\mathrm{N}} M_{0}(1+30 C)\right]\right\}}{70 R^{8}\left(R+6 C G_{\mathrm{N}} M_{0}\right)} \\
& +B\left(M_{0}, R\right)
\end{aligned}
$$


where $B$ is again an integration constant, which can be fixed like in the Newtonian case, so as to ensure that $M$ equals the total energy of the system,

$$
E \simeq M_{0}+U_{\mathrm{G}}+U_{\mathrm{B}}=M .
$$

The cumbersome expression (4.43) simplifies significantly if we consider a source with small compactness, i.e. $G_{\mathrm{N}} M_{0} / R \ll 1$ and $C \simeq 1+G_{\mathrm{N}} M_{0} / R$, which yields

$$
U_{\mathrm{B}}(R) \simeq B\left(M_{0}, R\right)-\frac{G_{\mathrm{N}} M_{0}^{2}}{5 R}-\frac{2 G_{\mathrm{N}}^{2} M_{0}^{3}}{35 R^{2}} .
$$

In this low compactness regime, $M$ is given in Eq. (4.21) and $U_{\mathrm{G}}$ by the first line of Eq. (A.5). Upon replacing these expressions in Eq. (4.44) then yields

$$
B\left(M_{0}, R\right) \simeq \frac{16 G_{\mathrm{N}} M_{0}^{2}}{5 R}+\frac{113 G_{\mathrm{N}}^{2} M_{0}^{3}}{70 R^{2}},
$$

and we finally end up with

$$
U_{\mathrm{B}}(R) \simeq \frac{3 G_{\mathrm{N}} M_{0}^{2}}{R}+\frac{109 G_{\mathrm{N}}^{2} M_{0}^{3}}{70 R^{2}} .
$$

As we pointed out in Section 4.3.1, our description let us also consider the opposite regime, i.e. the case of highly compact sources with $G_{\mathrm{N}} M_{0} / R \gg 1$. The corresponding baryonic potential energy then reads

$$
U_{\mathrm{B}}(R) \simeq B\left(M_{0}, R\right)-\frac{G_{\mathrm{N}}(1+21 C) M_{0}^{2}}{105 C R},
$$

and it is again straightforward to ensure that Eq. (4.44) is satisfied, by fixing the integration constant

$$
B\left(M_{0}, R\right) \simeq 2.22 \frac{G_{\mathrm{N}}^{2} M_{0}^{3}}{R^{2}},
$$

where we used the second expression in (A.5) for the gravitational energy $U_{\mathrm{G}}$ and Eq. (4.23) for $M$. It is finally immediate to write

$$
U_{\mathrm{B}}(R) \simeq 2.22 \frac{G_{\mathrm{N}}^{2} M_{0}^{3}}{R^{2}} .
$$

We would like to end this section with some remarks about the ratio $\Gamma_{0} \equiv p(0) / \rho(0)$. In General Relativity, it is well known [2] that, for any realistic spherical configuration of matter ${ }^{2}$, the pressure can be positive and non-singular in the origin only if the radius of the source is larger than the Buchdahl limit, that is for a source of size

$$
R>\frac{9}{4} G_{\mathrm{N}} M_{0} \equiv R_{\mathrm{BL}} .
$$

This is apparent from the expression of the pressure (D.3) obtained by solving the Tolman-Oppenheimer-Volkoff (TOV) equation (D.1) for a homogeneous density. If we instead calculate the above

\footnotetext{
${ }^{2}$ Non-outward increasing matter density and vanishing pressure at the surface.
} 
ratio for our case using the pressure from Eq. (4.42), also obtained for a homogeneous density, we find

$$
\Gamma_{0}=\frac{G_{\mathrm{N}} M_{0}}{2 R}\left[1+\frac{G_{\mathrm{N}} M_{0}}{5\left(R+6 C G_{\mathrm{N}} M_{0}\right)}\right] .
$$

For $R \simeq R_{\mathrm{BL}}$, and using the value of the constant $C$ from Eq. (4.25), this becomes

$$
\Gamma_{0}\left(R_{\mathrm{BL}}\right) \simeq 0.22,
$$

whereas $\Gamma_{0} \rightarrow \infty$ in the limit $R \rightarrow 0$. This means that the divergence in the pressure that one encounters for $R=R_{\mathrm{BL}}$ in the General Relativistic case has been pushed all the way down to $R \simeq 0$ in our model.

The above result leads us to remark that, although a static object with a uniform density is just a simple toy model, it can give us hints for more general arguments. For any density profile, the gravitational force is directed inwards and would collapse the mass towards the centre unless it were balanced by an equal and opposite pressure. For the homogeneous density we calculated this necessary pressure without discussing its origin or physical acceptability. In General Relativity, only for objects with radii larger than the Buchdahl limit, such as stars, this pressure could be provided by nuclear reactions or the Pauli exclusion principle: below the Buchdahl limit there exists no density profile which allows for a static configuration. This conclusion could only change if gravity departs from the General Relativistic description and provides itself an effective pressure directed outwards, like in the de Sitter space-time. In the corpuscular picture, the gravitons inside black holes form a Bose-Einstein condensate, whose equation of state resembles that of dark energy [11], and these gravitons are therefore natural candidates to provide an effective outward pressure. Moreover, such a large deviation of the ratio $\Gamma_{0}$ from General Relativity as the one we found above would imply that quantum effects have overcome the classical behaviour already at (relatively) large scales $R \simeq R_{\mathrm{BL}} \sim G_{\mathrm{N}} M_{0}$. Of course, one could then argue that this is only acceptable for masses $M_{0}$ sufficiently small, say around the Planck scale. However, one could also argue that the difference between the bootstrapped Newtonian description and General Relativity should become smaller if one included higher interaction terms in the Lagrangian (2.6), to the point that this difference might become phenomenologically negligible for astrophysical objects. Finally, we also recall that, unlike what happens in General Relativity, we assumed the potential energy density responsible for the pressure does not contribute a source term for the potential, and the addition of such a contribution to the Lagrangian (2.6) might again reduce the difference with respect to General Relativity.

\section{Conclusions and outlook}

In the previous sections, we have derived the potential satisfying the non-linear equation (2.7) generated by a spherically symmetric homogenous compact source. We have also obtained the necessary pressure (4.42) to keep the configuration static for sources of arbitrarily small radii, including values below the Buchdahl limit (4.50). Since this pressure turns out to be finite, unlike what one expects in General Relativity, it now seems appropriate to come back to our original motivation for constructing such a "bootstrapped" Newtonian ball and further clarify the possible connection with the corpuscular picture of black holes that we have mentioned in the Introduction and at the end of the last Section.

First of all, it is a theorem in General Relativity that, under rather general assumptions, systems that develop trapping surfaces will collapse into singularities [17]. This is what one expects would 
happen to a body that shrinks below the Buchdahl limit (4.50). On the other hand, a singularity is hardly acceptable in the quantum theory, just because of the Heisenberg uncertainty principle, and one could generically expect that the actual collapse of an astrophysical body will necessarily deviate from the General Relativistic description at some point. The crucial question is whether such deviations occur after the horizon has appeared, and they therefore remain hidden forever, or the quantum effects induce departures from General Relativity outside the gravitational radius which can therefore be observed. Many works have shown the existence of regular black hole solutions of modified gravitational equations which entail no significant departures from the corresponding General Relativistic space-times outside the (outer) horizon (for a review, see Ref. [18]). The corpuscular picture [11] instead assumes that black holes are fully quantum objects in order to give a consistent description of the Hawking evaporation and the possibility of observable consequences should therefore not be excluded.

In order to gain further insight into the above two possibilities, one should not forget about the matter that collapses and causes the emergence of the black hole geometry. In particular, one might wonder where this matter ends up in the corpuscular picture [8]. Clearly, if General Relativity remains a good theory of gravity up to extremely high energy densities, the collapsing matter should form a tiny ball with essentially no modifications of physics below the Planck energy scale. In the quantum corpuscular picture one could instead conceive the possibility that the collapsing matter occupies (in the quantum mechanical sense of the Compton length) a large volume inside the black hole and gives rise to an effective gravitational potential that differs significantly from the General Relativistic description. This is precisely the reason we developed the "bootstrapped" Newtonian picture of a homogeneous source, not to be taken as a model of phenomenological relevance for compact objects like neutron stars, but as a toy model of gravity tailored to further investigating this quantum picture of black holes. It is indeed rather likely that, in order to describe more accurately astrophysical compact objects, one would need to add more interacting terms to the Lagrangian (2.6), so that the gravitational potential outside the gravitational radius approaches the usual post-Newtonian expansion of the General Relativistic Schwarzschild metric.

Although the explicit quantum investigation of the bootstrapped Newtonian ball is left for a future work, we can here highlight a few relevant features. First of all, we have already noted that the (exact) vacuum solution $V_{\mathrm{c}}$ in Eq. (3.3) tracks the Newtonian behaviour, and its derivative therefore gives an attractive force for all (finite) values of $r>0$. This is in clear contrast with the weak field expansion in Eq. (3.4), which instead provides a repulsive force for $r \leq R_{\mathrm{H}}=2 G_{\mathrm{N}} M$, if one only includes the first post-Newtonian term $V_{\mathrm{PN}}$. In particular, one could apply a Newtonian argument to $V_{\mathrm{c}}$ and define the "horizon" as the place where the escape velocity of a particle subjected to it would equal the speed of light. This occurs for $2 V_{\mathrm{c}}\left(r_{\mathrm{H}}\right)=-1$, which yields

$$
r_{\mathrm{H}}=\frac{6 G_{\mathrm{N}} M}{3 \sqrt{3}-1} \simeq 1.4 G_{\mathrm{N}} M,
$$

or $r_{\mathrm{H}} \simeq 0.7 R_{\mathrm{H}}$. We remark once more that a source of size $R$ this small could not be studied in the weak field expansion to first post-Newtonian order, as that approximation does not hold for this range of the radial coordinate. In fact, it would also violate the Buchdahl limit (4.50) and could not be a stable configuration according to General Relativity.

Since black holes in General Relativity are regions of empty space with singular sources in the very centre, we can consider the case of a source with radius $R \ll r_{\mathrm{H}}$. Let us note that, at least for 
a homogeneous source, we always have $M>M_{0}$ and Eq. (4.23) then implies that the ratio

$$
\frac{M}{M_{0}} \simeq\left(\frac{6 G_{\mathrm{N}} M_{0}}{R}\right)^{1 / 2} \simeq\left(\frac{6 G_{\mathrm{N}} M}{R}\right)^{1 / 3} \gg 1,
$$

for $R \ll r_{\mathrm{H}}$. The area law of black holes [12] states that the mass $M$ should measure the number of gravitational degrees of freedom, which is also confirmed explicitly by the number of gravitons (1.2) in the corpuscular picture [11]. The above Eq. (5.2) therefore appears in line with the expectation that the number of gravitational degrees of freedom should largely overcome the number of matter degrees of freedom (proportional to $M_{0}$ ) in a black hole.

We have already argued in Section 4.5.2 why the mass parameter $M$ of the outer vacuum solution (3.3) should equal the total energy $E$ of the system. Moreover, the previous considerations naturally lead us to look more closely at the relation between $M$ and the proper mass $M_{0}$. In particular, if we add the proper energy $M_{0}$ to the gravitational potential energy $U_{\mathrm{G}}$ from Eq. (A.5), we find

$$
E_{0 \mathrm{G}} \equiv M_{0}+U_{\mathrm{G}} \simeq M_{0}\left(1-\alpha \frac{G_{\mathrm{N}} M_{0}}{R}-\beta \frac{G_{\mathrm{N}}^{2} M_{0}^{2}}{R^{2}}\right),
$$

where $\alpha$ and $\beta$ are numerical coefficients of order one, which can be estimated using the two approximations employed in Eq. (A.5) or computed numerically. We then find that the energy $E_{0 \mathrm{G}}$ is positive for low compactness sources with $G_{\mathrm{N}} M_{0} \ll R$, it vanishes for $G_{\mathrm{N}} M_{0} \simeq 0.6 R$ and becomes negative for very compact sources with $G_{\mathrm{N}} M_{0} \gg R$. For $R \simeq r_{\mathrm{H}}$, or

$$
G_{\mathrm{N}} M \simeq 0.7 r_{\mathrm{H}}
$$

one can solve the matching conditions (4.18) numerically and finds

$$
r_{\mathrm{H}} \simeq 2 G_{\mathrm{N}} M_{0} \text { and } \quad C \simeq 1.2 .
$$

Moreover, this value of $M_{0}$ is also close to the case that makes the energy (5.3),

$$
E_{0 \mathrm{G}}\left(r_{\mathrm{H}}\right)=M_{0}+U_{\mathrm{G}}\left(r_{\mathrm{H}}\right) \simeq 0 .
$$

We shall thus conclude by noting that the vanishing of $E_{0 \mathrm{G}}$ at (the threshold of) black hole formation appears as another form of the "maximal packing" condition at the heart of the corpuscular picture $[8,9,11]$. In fact, Eq. (5.6) is very close to the form of the maximal packing condition that is implemented in the quantum harmonic model of corpuscular black holes [16]. Whether this maximal packing conditions implies that the baryonic matter is completely delocalised inside the horizon like the gravitons requires a fully quantum treatment of the system and is left for future investigations.

\section{Acknowledgments}

The authors would like to thank M.F. de Laurentis, A. Giugno, A. Giusti, and J. Mureika for useful comments and suggestions. R.C. and M.L. are partially supported by the INFN grant FLAG. The work of R.C. has also been carried out in the framework of activities of the National Group of Mathematical Physics (GNFM, INdAM) and COST action Cantata. O.M. is supported by the grant Laplas V of the Romanian National Authority for Scientific Research. 


\section{A Potential energy}

The gravitational potential energy of the vacuum solution (3.3) can be easily computed from the effective Lagrangian (2.6) with $\rho=0$ and reads

$$
\begin{aligned}
U_{\mathrm{G}}^{+}\left[V_{\mathrm{c}}\right]=-L^{+}\left[V_{\mathrm{c}}\right] & =\frac{1}{2 G_{\mathrm{N}}} \int_{R}^{\infty} r^{2} \mathrm{~d} r(1-4 V)\left(V^{\prime}\right)^{2} \\
& =\frac{G_{\mathrm{N}} M^{2}}{2} \int_{R}^{\infty} \frac{\mathrm{d} r}{r^{2}}=\frac{G_{\mathrm{N}} M^{2}}{2 R},
\end{aligned}
$$

where $R$ can be simply viewed as a cut-off to regularise the result, in analogy with the Newtonian case.

If we instead consider a homogeneous ball in vacuum, the length $R$ naturally becomes the radius of the source. We again switch to dimensionless units and the total gravitational potential energy for the Newtonian solution (4.9) is found to be

$$
\begin{aligned}
\tilde{U}_{\mathrm{G}}\left[\tilde{V}_{\mathrm{N}}\right] & =-\tilde{L}\left[\tilde{V}_{\mathrm{N}}\right]=\frac{1}{2} \int_{0}^{\infty} \tilde{r}^{2} \mathrm{~d} \tilde{r}\left[\left(1-4 \tilde{V}_{\mathrm{N}}\right)\left(\tilde{V}_{\mathrm{N}}^{\prime}\right)^{2}+8 \pi \tilde{\rho} \tilde{V}_{\mathrm{N}}\left(1-2 \tilde{V}_{\mathrm{N}}\right)\right] \\
& =3 \tilde{M}_{0} \int_{0}^{1} \tilde{r}^{2} \mathrm{~d} \tilde{r} \tilde{V}_{\mathrm{N}}\left(1-2 \tilde{V}_{\mathrm{N}}^{2}\right)+\frac{1}{2} \int_{0}^{\infty} \tilde{r}^{2} \mathrm{~d} \tilde{r}\left[\left(1-4 \tilde{V}_{\mathrm{N}}\right)\left(\tilde{V}_{\mathrm{N}}^{\prime}\right)^{2}\right] \\
& =-\frac{3 \tilde{M}_{0}^{2}}{5}+\frac{51 \tilde{M}_{0}^{3}}{35}=-\frac{3 \tilde{M}_{0}^{2}}{5}\left(1-\frac{255 \tilde{M}_{0}}{105}\right)
\end{aligned}
$$

in which we recognize the standard (negative) Newtonian contribution obtained from the first line in Eq. (2.4), that is

$$
\tilde{U}_{\mathrm{N}}=\frac{\tilde{M}_{0}^{2}}{4} \int_{0}^{1} \tilde{r}^{2} \mathrm{~d} \tilde{r} \tilde{V}_{\mathrm{N}}=-\frac{3 \tilde{M}_{0}^{2}}{5} .
$$

Like for the exact vacuum solution (3.3), the last integral in the second line of Eq. (A.2) yields a positive contribution, which is however overcome by the negative Newtonian energy and its (still negative) correction arising where $\tilde{\rho} \neq 0$.

Given the correct solution to the effective equation (4.4) is very close to the Newtonian expression (4.8) inside the ball, we can estimate the contribution to the potential energy from the region inside the source as ${ }^{3}$

$$
\begin{aligned}
\tilde{U}_{\mathrm{G}}^{-}\left[\tilde{V}_{\mathrm{c}}\right] \simeq-L^{-}\left[\tilde{V}_{\mathrm{N}}\right] & =\frac{1}{2} \int_{0}^{1} \tilde{r}^{2} \mathrm{~d} \tilde{r}\left[\left(1-4 \tilde{V}_{\mathrm{N}}\right)\left(\tilde{V}_{\mathrm{N}}^{\prime}\right)^{2}+6 \tilde{M}_{0} \tilde{V}_{\mathrm{N}}\left(1-2 \tilde{V}_{\mathrm{N}}\right)\right] \\
& \simeq-\frac{3 \tilde{M}_{0}^{2}}{2}\left[C-\frac{4}{15}+\tilde{M}_{0}\left(\frac{5}{21}-\frac{24}{15} C+3 C^{2}\right)\right] .
\end{aligned}
$$

We can finally estimate the total gravitational energy of the homogeneous ball by adding together Eq. (A.1) for the outer vacuum and the contribution (A.4) from the interior, which yields

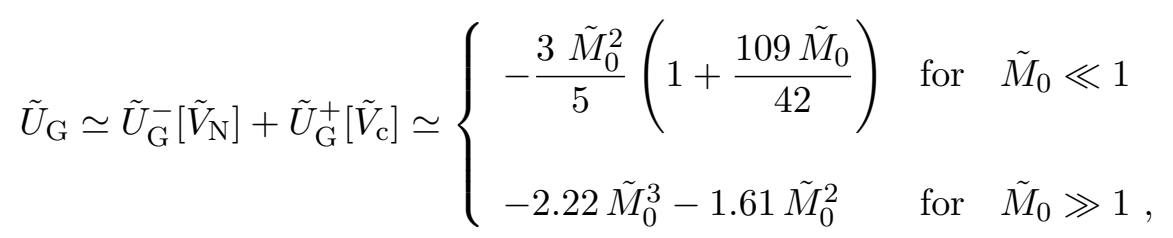

\footnotetext{
${ }^{3}$ We also checked that using the approximation (4.14) with Eq. (4.16) does not alter the result significantly.
} 


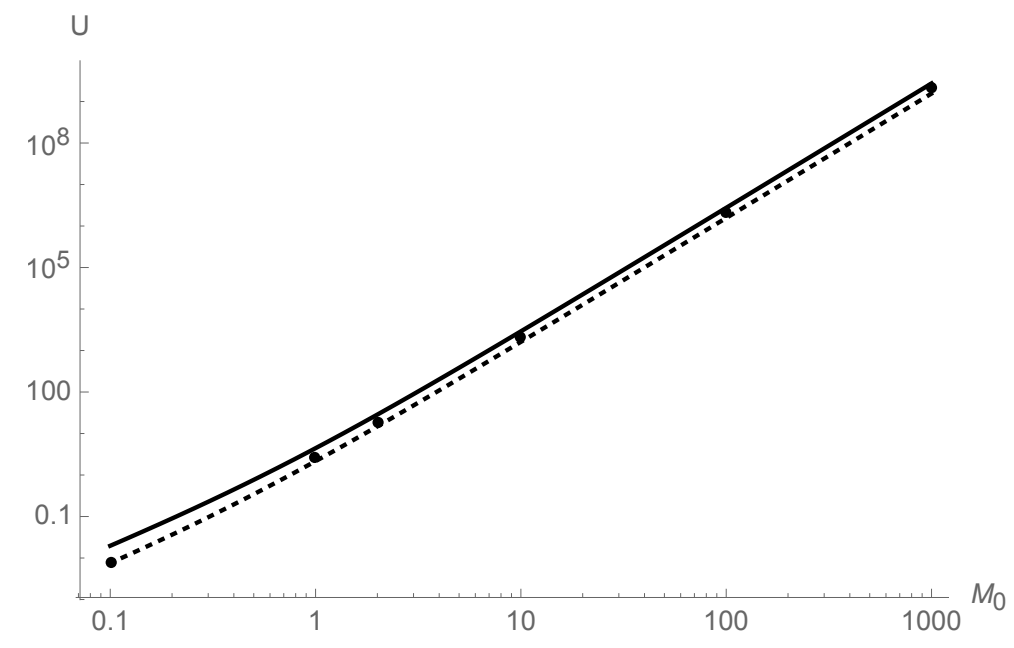

Figure 7: Gravitational potential energy $\tilde{U}_{\mathrm{G}}$ evaluated numerically (dots) vs the analytical approximation (A.5) for $\tilde{M}_{0} \gg 1$ (solid line) vs the analytical approximation (A.5) for $\tilde{M}_{0} \ll 1$ (dotted line).

in which we used Eqs. (4.21) and (4.22) for $\tilde{M}_{0} \ll 1$ and Eqs. (4.23) and (4.25) for $\tilde{M}_{0} \gg 1$.

The gravitational energy $\tilde{U}_{\mathrm{G}}$ can also be computed numerically for given values of $\tilde{M}_{0}$, and compared with the above analytical approximations. From the plot in Fig. 7, it appears that both approximate expressions reproduce the numerical results fairly well.

\section{B Junction conditions}

Because the shift symmetry is lost, we cannot just match the outer potential (3.3) with just any interior. It is then useful to analyse the matching conditions at $r=R$. This can be done as usual by integrating Eq. (2.7) on a shell of thickness $0<2 \epsilon \ll R$ around $r=R$,

$$
0=\int_{R-\epsilon}^{R+\epsilon} r^{2} \mathrm{~d} r\left[(1-4 V)\left(\triangle V-4 \pi G_{\mathrm{N}} \rho\right)-2\left(V^{\prime}\right)^{2}\right] \equiv I_{1}+I_{2}+I_{3}
$$

For the first term we obtain

$$
\begin{aligned}
I_{1} & =\int_{R-\epsilon}^{R+\epsilon} \mathrm{d} r(1-4 V)\left(r^{2} V^{\prime}\right)^{\prime} \\
& =\left[(1-4 V)\left(r^{2} V^{\prime}\right)\right]_{R-\epsilon}^{R+\epsilon}+4 \int_{R-\epsilon}^{R+\epsilon} r^{2} \mathrm{~d} r\left(V^{\prime}\right)^{2},
\end{aligned}
$$

so that

$$
I_{1}+I_{3}=\left[(1-4 V)\left(r^{2} V^{\prime}\right)\right]_{R-\epsilon}^{R+\epsilon}+2 \int_{R-\epsilon}^{R+\epsilon} r^{2} \mathrm{~d} r\left(V^{\prime}\right)^{2} .
$$

If we assume $\rho$ and $V$ do not diverge at $r=R$, the second term vanishes for $\epsilon \rightarrow 0$,

$$
I_{2}=4 \pi G_{\mathrm{N}} \int_{R-\epsilon}^{R+\epsilon} r^{2} \mathrm{~d} r(1-4 V) \rho \underset{\epsilon \rightarrow 0}{\rightarrow} 0
$$


and one is left with

$$
0=\left[(1-4 V)\left(r^{2} V^{\prime}\right)\right]_{R-\epsilon}^{R+\epsilon}+2 \int_{R-\epsilon}^{R+\epsilon} r^{2} \mathrm{~d} r\left(V^{\prime}\right)^{2},
$$

which is satisfied for $\epsilon \rightarrow 0$ if $V^{\prime}$ is continuous across $r=R$.

\section{Anti-de Sitter potential?}

Eq. (2.7) with the homogeneous density in Eq. (4.1) is solved exactly for $0 \leq r<R$ by

$$
V_{\mathrm{c}}=\frac{1}{4}+\frac{3 G_{\mathrm{N}} M_{0}}{8 R^{3}} r^{2},
$$

which is clearly positive everywhere, and cannot be matched with a negative outer potential at $r=R$. This solution could however be considered as a "cosmological" solution similar to the anti-de Sitter space.

\section{Comparison with TOV}

It is instructive to compare our result (4.42) for the pressure with the expectation obtained by solving the TOV equation for a homogeneous source. The standard TOV equation relating the pressure and energy density reads [2]

$$
\frac{\mathrm{d} p(r)}{\mathrm{d} r}=\frac{[\rho+p(r)] G_{\mathrm{N}}\left[2 m(r)+8 \pi p(r) r^{3}\right]}{2 r\left[2 G_{\mathrm{N}} m(r)-r\right]},
$$

where $m(r)$ is the mass function

$$
m(r)=4 \pi \int_{0}^{r} \mathrm{~d} r^{\prime} \rho\left(r^{\prime}\right) r^{2} .
$$

Its solution for the homogeneous density (4.1) can be found in exact form after requiring that $p_{\text {TOV }}(R)=0$. This yields

$$
\begin{aligned}
p_{\mathrm{TOV}}(r) & =\rho(r) \frac{\sqrt{R^{3}-2 G_{\mathrm{N}} M_{0} r^{2}}-R \sqrt{R-2 G_{\mathrm{N}} M_{0}}}{3 R \sqrt{R-2 G_{\mathrm{N}} M_{0}}-\sqrt{R^{3}-2 G_{\mathrm{N}} M_{0} r^{2}}} \\
& =\frac{3 M_{0}\left(\sqrt{R^{3}-2 G_{\mathrm{N}} M_{0} r^{2}}-R \sqrt{R-2 G_{\mathrm{N}} M_{0}}\right)}{4 \pi R^{3}\left(3 R \sqrt{R-2 G_{\mathrm{N}} M_{0}}-\sqrt{R^{3}-2 G_{\mathrm{N}} M_{0} r^{2}}\right)} .
\end{aligned}
$$

This TOV pressure can easily be compared to our Post-Newtonian result from Eq. (4.42) in the small compactness regime $G_{\mathrm{N}} M_{0} \ll R$, that is far from the Buchdahl limit. Considering the first two leading terms, the TOV pressure is approximately equal to

$$
p_{\mathrm{TOV}}(r) \simeq \frac{3\left(R^{2}-r^{2}\right) G_{\mathrm{N}} M_{0}^{2}}{8 \pi R^{6}}\left(1+\frac{8 G_{\mathrm{N}} M_{0}}{3 R}\right),
$$



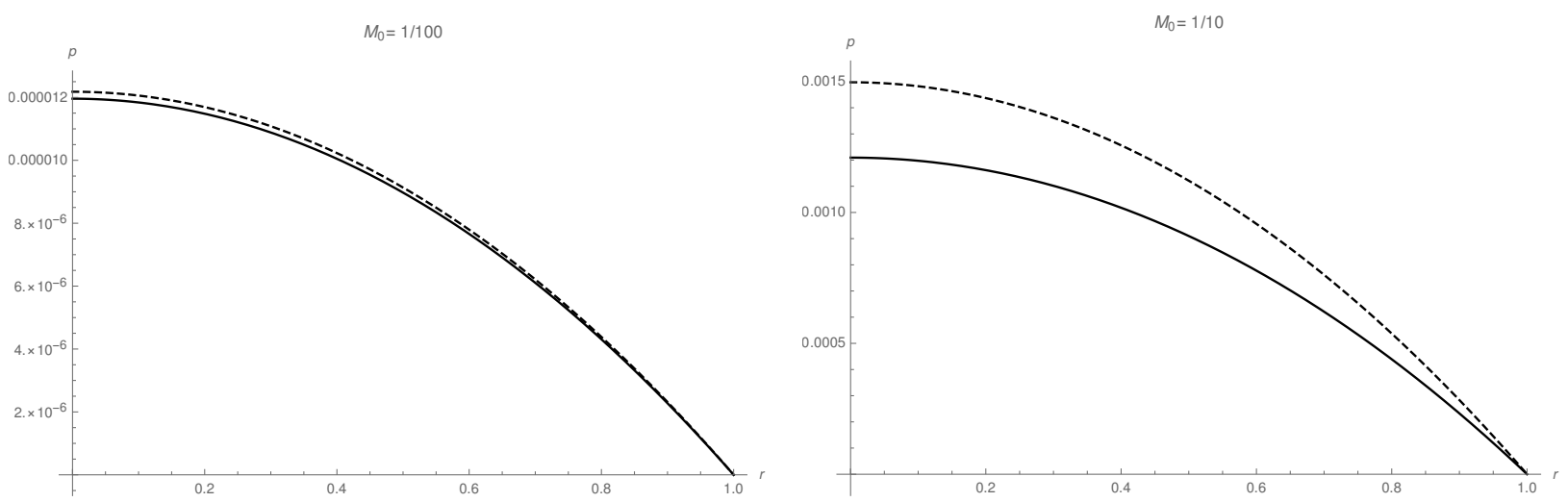

Figure 8: Numerical solution (solid line) vs $p_{\text {TOV }}$ (dashed line) for $\tilde{M}_{0}=1 / 100$ (left panel) and $\tilde{M}_{0}=1 / 10$ (right panel).

while the Post-Newtonian pressure from Eq. (4.42) is approximately

$$
p(r) \simeq \frac{3\left(R^{2}-r^{2}\right) G_{\mathrm{N}} M_{0}^{2}}{8 \pi R^{6}}\left[1+\frac{\left(R^{2}+r^{2}\right) G_{\mathrm{N}} M_{0}}{5 R^{3}}\right] .
$$

Both the lowest order term of the TOV pressure and the one of the Post-Newtonian pressure are equal to what one calculates in the Newtonian case (4.35). We can also remark that the next-toleading order contributions are much smaller. Therefore, as expected, in this limit the pressure inside the objects obtained in our model can be well approximated by the Newtonian pressure.

A comparison between the TOV solution and the numerical evaluation of the pressure within our model is displayed in Fig. 8. The plots also show that the two are in good agreement as long as the source is sufficiently less compact than the Buchdahl limit (4.50). This is not surprising, since the pressure (4.42) remains finite for any $0 \leq r<R$, whereas $p_{\mathrm{TOV}}(0)$ in Eq. (D.3) diverges for $R \rightarrow R_{\mathrm{BL}}^{+}$

\section{References}

[1] S. Weinberg, "Gravitation and Cosmology: Principles and Applications of the General Theory of Relativity," (Wiley and Sons, New York, 1972)

[2] H. Stephani "Relativity: An Introduction to Special and General Relativity" (Cambridge University Press, Cambridge, 2004)

[3] S. Deser, Gen. Rel. Grav. 1 (1970) 9 [gr-qc/0411023]; S. Deser, Gen. Rel. Grav. 42 (2010) 641 [arXiv:0910.2975 [gr-qc]].

[4] R. M. Wald, Phys. Rev. D 33 (1986) 3613; C. Cutler and R. M. Wald, Class. Quant. Grav. 4 (1987) 1267; R. M. Wald, Class. Quant. Grav. 4 (1987) 1279.

[5] K. Heiderich and W. Unruh, Phys. Rev. D 38 (1988) 490; Phys. Rev. D 42 (1990) 2057.

[6] M. P. Hertzberg, "Gravitation, Causality, and Quantum Consistency," arXiv:1610.03065 [hepth]. M. P. Hertzberg and M. Sandora, JHEP 1709 (2017) 119 [arXiv:1702.07720 [hep-th]]. 
[7] D. Bai and Y. H. Xing, "Higher Derivative Theories for Interacting Massless Gravitons in Minkowski Spacetime," arXiv:1610.00241 [hep-th].

[8] R. Casadio, A. Giugno and A. Giusti, Phys. Lett. B 763 (2016) 337 [arXiv:1606.04744 [gr-qc]]

[9] R. Casadio, A. Giugno, A. Giusti and M. Lenzi, Phys. Rev. D 96044010 (2017) [arXiv:1702.05918 [gr-qc]].

[10] G. Schäfer and P. Jaranowski, "Hamiltonian formulation of general relativity and postNewtonian dynamics of compact binaries," arXiv:1805.07240 [gr-qc].

[11] G. Dvali and C. Gomez, JCAP 01 (2014) 023; "Black Hole's Information Group", arXiv:1307.7630; Eur. Phys. J. C 74 (2014) 2752 [arXiv:1207.4059 [hep-th]]; Phys. Lett. B 719 (2013) 419 [arXiv:1203.6575 [hep-th]]; Phys. Lett. B 716 (2012) 240 [arXiv:1203.3372 [hep-th]]; Fortsch. Phys. 61 (2013) 742; G. Dvali, C. Gomez and S. Mukhanov, "Black Hole Masses are Quantized," arXiv:1106.5894 [hep-ph].

[12] J.D. Bekenstein, Phys. Rev. D 7 (1973) 2333.

[13] N. Dadhich, Curr. Sci. 76 (1999) 831 [gr-qc/9705037].

[14] N. Dadhich, Curr. Sci. 109 (2015) 260 [arXiv:1206.0635 [gr-qc]].

[15] R.L. Arnowitt, S. Deser and C.W. Misner, Phys. Rev. 116 (1959) 1322.

[16] R. Casadio and A. Orlandi, JHEP 1308 (2013) 025 [arXiv:1302.7138 [hep-th]]; R. Casadio, A. Giugno, A. Giusti and O. Micu, Eur. Phys. J. C 77 (2017) no.5, 322 [arXiv:1701.05778 [gr-qc]|.

[17] S. W. Hawking and G. F. R. Ellis, "The Large Scale Structure of Space-Time," (Cambridge University Press, Cambridge, 1973)

[18] P. Nicolini, Int. J. Mod. Phys. A 24 (2009) 1229 [arXiv:0807.1939 [hep-th]]. 